\title{
O Impacto da Monitorização Eletrónica nas Infeções Associadas aos Cuidados de Saúde: O Papel da Plataforma HViTAL
}

\author{
The Impact of Electronic Monitoring on Healthcare \\ Associated Infections: The Role of the HViTAL Platform
}

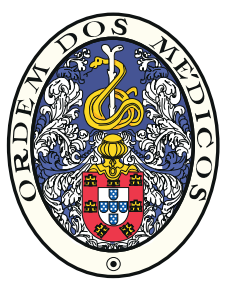

\author{
Rita Fontes de OLIVEIRA $\triangle^{1,2}$, Lídia CASTRO ${ }^{3}$, José Pedro ALMEIDA ${ }^{4}$, Carlos ALVES ${ }^{5}$, António FERREIRA ${ }^{6}$ \\ Acta Med Port 2016 Nov;29(11):734-741 - http://dx.doi.org/10.20344/amp.7388
}

\section{RESUMO}

Introdução: Em Portugal, 9,8\% dos doentes internados adquiriram infeções associadas aos cuidados de saúde, correspondendo a uma prevalência de cerca $11,7 \%$. O Centro Hospitalar de São João desenvolveu uma plataforma de business intelligence capaz de Vlgiar (os utentes), moniTorizar (o estado clínico) e ALertar (o profissional de saúde): HViTAL. Este estudo tem como objectivo avaliar o impacto da monitorização eletrónica nas infeções associadas aos cuidados de saúde desde o ano da implementação do HViTAL.

Material e Métodos: Avaliaram-se os dados relativos a janeiro 2008 (data a partir da qual há registos informatizados) até dezembro de 2011, comparando-os com os dados posteriores, aqueles correspondentes a janeiro de 2012 (data de implementação do HViTAL) até 19 de outubro de 2015.

Resultados: Observou-se uma tendência para o aumento dos parâmetros de infecção no período 2008 - 2011. No período correspondente a janeiro de 2012 e outubro de 2015, todos os parâmetros que constituem o indicador de infeção (previsto na contratualização) revelaram uma evolução linear negativa.

Discussão: Os resultados são muito sugestivos de que o HVITAL poderá ter tido impacto na melhoria dos parâmetros associados às infeções associadas aos cuidados de saúde. Houve uma tendência crescente na aplicação de medidas básicas de controlo de infecção desde 2005, com ações de sensibilização dos profissionais de saúde, facto que, embora não analisado neste report, poderá também ter contribuído para a melhoria observada. O nosso estudo não incluiu outras variáveis tais como investimento em capital humano.

Conclusão: Houve uma inequívoca melhoria em todos os domínios que caracterizam as infeções associadas aos cuidados de saúde, sugerindo um impacto positivo da introdução do HVITAL.

Palavras-chave: Controle de Infecções; Infecção Hospitalar; Informática Médica; Registos Eletrónicos de Saúde.

\section{ABSTRACT}

Introduction: In Portugal, 9.8\% of patients admitted were inflicted with healthcare associated infections, corresponding to a prevalence of $11.7 \%$. The Hospital de São João has developed a business intelligence platform able to supervise (the patients), monitor (the clinical condition) and notify (the healthcare personnel): HViTAL. This study aims to assess the impact of electronic monitoring on healthcare associated infections since the year of HViTAL implementation.

Material and Methods: We evaluated data since January 2008 (moment from which computerized records exist) until December 2011, comparing them with subsequent data, those corresponding to January 2012 (implementation date of HViTAL) until 19 October 2015. Results: There was an upward trend of infection parameters in the 2008 - 2011 period. Since January 2012 and October 2015 , all parameters of the infection indicator showed a negative linear trend.

Discussion: The results are very suggestive that the HVITAL may have had an impact on improving parameters associated to healthcare associated infections. Basic measures of infection control were highlighted since 2005, with an increasing number of health professional awareness campaigns, a fact which, although not analyzed in this report, may also have contributed to the observed improvement. Our study did not include other variables such as investment in human capital.

Conclusion: There was a clear improvement in all areas characterizing the healthcare associated infections, with obvious positive impact with the introduction of HViTAL.

Keywords: Cross Infection; Electronic Health Records; Infection Control; Medical Informatics.

\section{INTRODUÇÃO}

A nível europeu, os estudos apontam para uma prevalência de $5 \%$ a $10 \%$ de infeções em doentes hospitalizados. ${ }^{1}$ Nos países em desenvolvimento o risco é duas a vinte vezes maior e a prevalência dos doentes infetados excede os $25 \% .^{2}$ O seu impacto implica hospitalização prolongada, aparecimento de comorbilidades, aumento das taxas de mortalidade e aumento da resistência a antimicrobianos. Do ponto de vista económico, acarretam um esforço financeiro adicional devido aos custos elevados que Ihes são inerentes. ${ }^{1}$

Os Centers for Disease Control and Prevention (CDC) definem a infeção associada aos cuidados de saúde (IACS)

\footnotetext{
1. Departamento de Medicina Geral e Familiar. Unidade de Saúde Familiar de São João. Porto. Portugal.

2. Departamento de Ciências Sociais e Saúde. Faculdade de Medicina. Universidade do Porto. Porto. Portugal.

3. Serviço de Controlo de Gestão. Centro Hospitalar de São João. Porto. Portugal.

4. Projeto Clinical Intelligence. Centro Hospitalar de São João. Porto. Portugal.

5. Unidade de Prevenção e Controlo da Infecção e Resistência aos Antimicrobianos. Centro Hospitalar de São João. Porto. Portugal

6. Conselho de Administração. Hospital de São João. Porto. Portugal.

$\triangle$ Autor correspondente: Rita Fontes de Oliveira. ritafontesoliveira@gmail.com

Recebido: 02 de janeiro de 2016 - Aceite: 02 de setembro de 2016 | Copyright @ Ordem dos Médicos 2016
} 
como uma situação sistémica ou localizada resultante de uma reação adversa à presença de um agente (ou agentes) infecioso ou da sua toxina (ou toxinas). Para o seu diagnóstico, a infecção não pode estar presente, ou em incubação, à data em que ocorre a admissão na unidade hospitalar. ${ }^{3}$

Os registos médicos eletrónicos (RME) são registos sobre a monitorização dos utentes que podem ser processados por um computador. Com o aumento da adoção de sistemas de RME cada vez mais sofisticados e custo-efetivos, tem-se verificado que esta nova ferramenta auxilia na economia de tempo, tanto no âmbito da vigilância das IACS como na gestão de casos clínicos. ${ }^{4}$

Há uma necessidade de implementação de medidas precisas, oportunas e eficientes de monitorização e gestão de eventos adversos em hospitais. Os sistemas de informação são um requisito essencial para avaliar a eficácia das intervenções preventivas e para acompanhar os progressos na segurança do paciente ao longo do tempo. ${ }^{5} \mathrm{~A}$ necessidade da sua implementação é uma realidade. ${ }^{6}$

No Relatório do Inquérito de Prevalência de Infecção de 2010, criado no âmbito do Programa Nacional de Prevenção e Controlo das IACS, participaram 97 hospitais (21 011 doentes). Em 9,8\% dos doentes foram identificadas IACS, correspondendo a uma prevalência de $11,7 \% .{ }^{7}$ No Inquérito de 2012 sobre a Prevalência de Infeção Adquirida no Hospital e do uso de Antimicrobianos nos Hospitais Portugueses, a taxa global de prevalência de IACS foi de $10,6 \%$ $(10,1$ - 11,0$)$, sendo de $12,4 \%$ nos homens e $8,8 \%$ nas muIheres, valores superiores à média europeia. Verificou-se, ainda neste estudo, uma elevada taxa de resistências aos antimicrobianos. ${ }^{8}$

Muitas IACS poderão ser prevenidas. No entanto, desconhece-se a proporção de IACS potencialmente evitáveis. ${ }^{6}$

Do ponto de vista da estrutura, é necessário redesenhar a constituição, papel e implantação das Comissões de Controlo de Infeção e das Comissões de Antibióticos. A participação empenhada de médicos, enfermeiros e farmacêuticos é fundamental. ${ }^{7}$ Nos últimos anos têm surgido estratégias mais seguras de realização de procedimentos e de utilização de dispositivos invasivos. As principais medidas de prevenção têm por base precauções básicas e isolamento, mas, também, assentam no uso apropriado de antimicrobianos. ${ }^{9-11}$ Deste modo, dever-se-á dar especial enfoque a três vertentes: a prevenção e controlo da infeção, a prevenção das resistências e o uso racional de antimicrobianos.

O Centro Hospitalar São João (CHSJ) desenvolveu uma plataforma tecnológica capaz de recolher, armazenar e analisar, em tempo real, os milhares de dados que a instituição produz. Esta plataforma de business intelligence (BI) tem dado um apoio fundamental à gestão e operação do CHSJ no alcance de resultados de excelência, na delineação de estratégias capazes de responder às necessidades da instituição e no benchmarking nacional e internacional.

Esta plataforma interliga e correlaciona toda a informação relevante, permitindo a pronta caracterização clínica, epidemiológica e financeira da população de doentes que recorre ao hospital. Através destes dados, é possível, entre outras variáveis, qualificar esta população, perceber de que patologias são portadores e consultar exames complementares de diagnóstico e terapêuticos (MCDT's) efetuados.

Atualmente o CHSJ, em conjunto com o DevScope ${ }^{\circledR}$, está a potenciar as capacidades desta tecnologia de modo a colocá-la ainda mais ao serviço dos seus utentes e dos seus profissionais. A plataforma HViTAL está preparada para agregar toda a informação, fazendo uma monitorização de parâmetros fisiológicos e resultados laboratoriais dos doentes. Tem como objetivo detetar padrões relativos ao estado clínico do utente, estimando índices de risco ou de morte de cada individuo que dá entrada no hospital. $\mathrm{O}$ sistema está preparado para identificar, categorizar e enviar alertas relativos a doentes em risco para as equipas clínicas responsáveis. Todos os dados relativos às infeções, como por exemplo agentes responsáveis, testes de sensibilidade a antibióticos, duração da antibioterapia, entre outros, são disponibilizados informaticamente ao coordenador da unidade de prevenção e controlo da infecção e resistência aos antimicrobianos (UPCIRA) que agilizará intervenções posteriores, incluindo a sensibilização dos profissionais de saúde. Acresce que cada médico individualmente recebe alertas no seu perfil informático relativos a alguns parâmetros clínicos dos doentes pelos quais é responsável (ex: duração da antibioterapia). Desta forma, garante-se que as medidas tomadas em áreas como o risco de deterioração clínica em pacientes internados, a infeção hospitalar e o consumo de antibióticos sejam cada vez mais eficazes.

Com base nestes pressupostos, pretende-se com este estudo avaliar o impacto da monitorização eletrónica nas IACS, desde o ano da implementação do HViTAL no CHSJ. O presente trabalho visa avaliar a evolução dos resultados relativos à infeção e uso de antimicrobianos, previsto no contrato-programa da ARS Norte desde 2012, no sentido de verificar se houve melhoria nos seus diferentes domínios após a introdução dos novos sistemas de informação.

\section{MATERIAL E MÉTODOS}

As duas populações em estudo são constituídas pelos utentes do CHSJ. Avaliámos 969246 episódios (urgência e consulta), dos quais originaram 343878 internamentos. Os dados encontram-se inseridos na plataforma HViTAL após anonimização.

Estudámos os dados relativos a janeiro 2008 até dezembro de 2011, comparando-os com aqueles correspondentes a janeiro de 2012 (data de implementação do HViTAL) até 19 de outubro de 2015.

Entre 2008 e 2011, analisaram-se os seguintes parâmetros normalmente relacionados com as infeções nosocomiais (variáveis dependentes primárias): i) dose definida diária (DDD) antibióticos (AB); ii) DDD carbapenemos; iii) DDD quinolonas; iv) dias $A B+$ dias totais de internamento; v) $A B>8 d$ + dias totais de internamento; vi) litros de solução antisséptica de base alcoólica (SABA) + número total de doentes. 
Para além dos supracitados, com a introdução do HViTAL em 2012, a Unidade de Prevenção e Controlo de Infeção e Resistências aos Antimicrobianos (UPCIRA) do CHSJ, utiliza para avaliar o indicador de infeção e antimicrobianos contratualizado com a ARS Norte outros parâmetros como ${ }^{11,12}$ : i) MMR (microrganismos multi-resistentes) + número total de doentes; ii) Staphylococcus aureus resistente à meticilina (MRSA) + número total de doentes; iii) MRSA + número total de doentes; iv) bacteriémias nosocomiais (BN) MRSA + número total de doentes.

Os dados dos diferentes programas informáticos do CHSJ estão condensados na plataforma de HViTAL, sendo recolhidos de forma automática na sua interface. Após a recolha dos dados, através do programa SPSS ${ }^{\circledR}$, estes foram
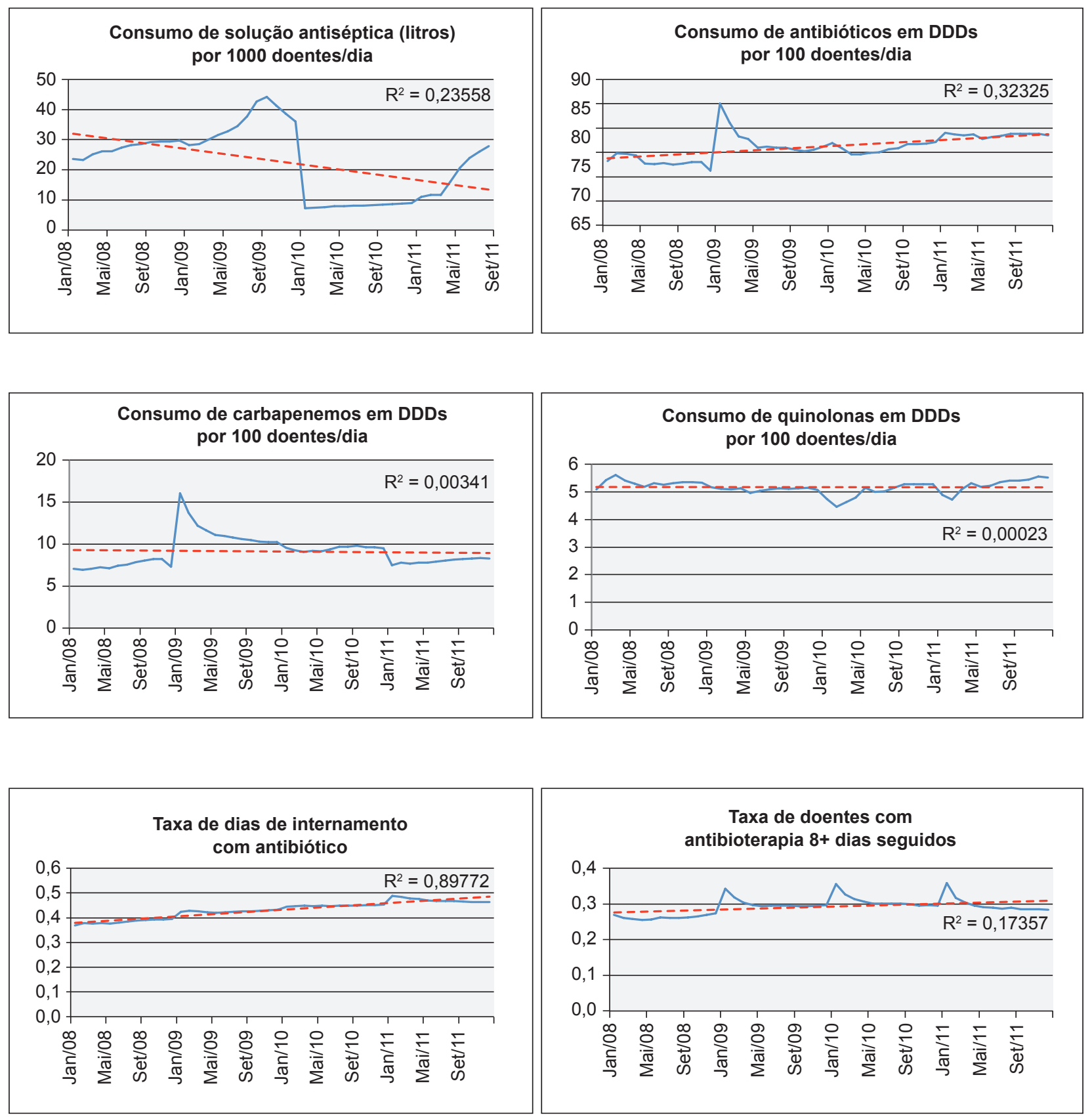

Figura 1 - Tendência dos parâmetros no período 2008 - 2011 analisados e tratados através do Excel: MAC ${ }^{\circledR}$.

Do ponto de vista da estatística inferencial, optou-se por ajustar os dados recolhidos a modelos lineares, apreciando o declive das retas e aferindo a qualidade do ajuste com os coeficientes de determinação. Em dois casos optou-se por um modelo de regressão polinomial de grau superior a 1 , devido à não linearidade dos gráficos respetivos. A qualidade do ajuste foi aferida, em cada caso, pelo coeficiente de determinação respetivo $\left(R^{2}\right)$, idealmente igual a 1 . vel de confiança de $0,1 \%$, a hipótese de a situação inicial de janeiro de 2008 e janeiro 2012, tida como valor de referência para população de leituras, não ter sofrido alterações. Dado o elevado número de observações, foram

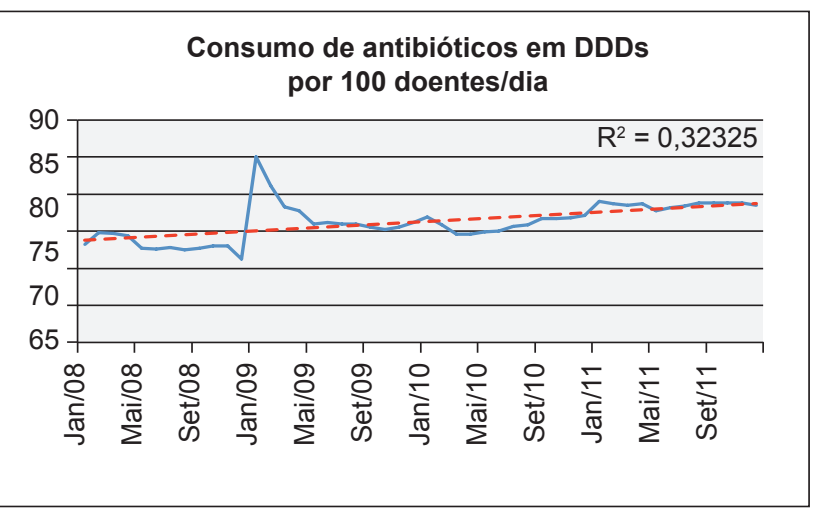

Foi também testada, para cada parâmetro e com ní- 
realizados testes $Z$ para a média. Considerou-se um $p \leq$ 0,05 como estatisticamente significativo. Foram efetuados teste $Z$ à diferença de valores esperados de duas populações com amostras independentes grandes (2008 - 2011 e 2012 - 2015).

\section{RESULTADOS}

A tendência para aumento dos parâmetros no período 2008 - 2011, ainda que ligeira em alguns deles, é evidente (Fig. 1). O consumo de solução antissética revela também tendência de crescimento neste período, apesar da diminuição da sua utilização entre os meses de janeiro de 2010 e abril de 2011 (Tabela 1). Com a exceção do consumo de solução antissética (litros) por 1000 doentes/dia, em que não se conseguiu rejeitar a hipótese nula, em todos os outros testes de hipóteses ao valor médio da amostra, verificou-se que os valores $p$ são praticamente nulos em cada deles, pelo que se rejeita a hipótese nula de o valor de referência se ter mantido (Tabela 1).

No segundo período do estudo, correspondente a janeiro de 2012 e outubro de 2015, todos os parâmetros do indicador de infeção revelaram uma evolução linear negativa desde 2012, ano de implementação do BI (Fig. 2), com exceção do consumo de solução antissética (litros) por 1000 doentes/dia que apresentou uma evolução linear positiva (Fig. 2). Foi o consumo de antibióticos em DDD por 100 doentes/dia o parâmetro que diminuiu de forma quadrática mais acentuada (Fig. 2). As bacteriemias nosocomiais a MRSA por 1000 doentes/dia não apresentaram tendência evidente (Fig. 2). De notar a oscilação de valores no gráfico relativo às bacteriemias nosocomiais MRSA por 1000 doentes/dia (Fig. 2), correspondente ao facto de afetarem um número muito reduzido de doentes relativamente ao total de indivíduos internados, pelo que se fazem notar oscilações de maior amplitude. Em relação aos testes de hipóteses ao valor médio da amostra, verificou-se que os valores $p$ muito inferiores a 0,05 em cada um dos testes, pelo que em todos se rejeita, claramente, a hipótese nula de o valor de referência se ter mantido (Tabela 2).

Quando comparados os dados das duas populações, as hipóteses de as médias não se alterarem são rejeitadas (Tabela 3). Excetuam-se os parâmetros taxa de dias de internamento com antibiótico e taxa de doentes com antibioterapia com mais de oito dias seguidos, onde as médias são idênticas. Este facto pode estar relacionado com o aumento no primeiro período e uma diminuição no segundo período (Fig. 3).

\section{DISCUSSÃO}

A tendência para o aumento dos parâmetros no período 2008 - 2011, ainda que ligeira em alguns deles, é clara. Com a introdução de HViTAL em 2012, todos os parâmetros do indicador de infecção revelaram uma evolução linear negativa desde então. Os resultados sugerem que o HVITAL poderá ter tido impacto na melhoria dos parâmetros associados às IACS.

As medidas básicas de controlo de infeção foram intervenções priorizadas desde 2005 , sendo alvo crescente de ações de sensibilização dos profissionais de saúde, facto que, embora não analisado neste report, poderá também ter contribuído para a melhoria observada. O nosso estudo não incluiu outras variáveis como investimento em capital humano.

A taxa de dias de internamento com antibiótico também foi considerada para colmatar as limitações das medidas de DDD. ${ }^{13}$ As doses recomendadas pela OMS por DDD podem não corresponder às doses de otimização do antibiótico e, portanto, podem não ser reflexo dos dias de terapia. Não menos importante foi a inclusão no indicador de infeção, da taxa de doentes com antibioterapia com mais de oito dias seguidos. O número de dias sob administração de antibióticos foi, na maioria dos casos, limitado a um número inferior a oito. ${ }^{11}$

Uma das limitações do nosso estudo é o facto da melhoria dos parâmetros poder ser devida ao reforço das medidas básicas de controlo de infeção, incluindo a

Tabela 1 - Teste de Hipóteses para a média (dados de 2008 a 2011)

\begin{tabular}{|c|c|c|c|c|c|c|c|}
\hline & $\mu_{0}{ }^{(1)}$ & Média e DP & $\mathrm{n}^{(2)}$ & $\boldsymbol{\alpha}^{(3)}$ & Z observado & Valor $p$ & $\begin{array}{l}\text { Resultado do } \\
\text { teste }\end{array}$ \\
\hline $\begin{array}{l}\text { Consumo de antibióticos em } \\
\text { DDDs por } 100 \text { doentes/dia }\end{array}$ & 73,23 & $76,27(2,59)$ & 48 & $0,1 \%$ & 8,14 & aprox. zero & rejeitar $\mathrm{HO}$ \\
\hline $\begin{array}{l}\text { Consumo de carbapenemos em } \\
\text { DDDs por } 100 \text { doentes/dia }\end{array}$ & 7,07 & $9,11(1,81)$ & 48 & $0,1 \%$ & 7,81 & aprox. zero & rejeitar $\mathrm{HO}$ \\
\hline $\begin{array}{l}\text { Consumo de quinolonas em } \\
\text { DDDs por } 100 \text { doentes/dia }\end{array}$ & 5,09 & $5,17(0,24)$ & 48 & $1,0 \%$ & 2,47 & $0,7 \%$ & rejeitar $\mathrm{HO}$ \\
\hline $\begin{array}{l}\text { Taxa de dias de internamento } \\
\text { com antibiótico }\end{array}$ & 0,37 & $0,43(0,03)$ & 48 & $0,1 \%$ & 13,19 & aprox. zero & rejeitar $\mathrm{HO}$ \\
\hline $\begin{array}{l}\text { Taxa de doentes com } \\
\text { antibioterapia } 8+\text { dias seguidos }\end{array}$ & 0,27 & $0,29(0,02)$ & 48 & $0,1 \%$ & 6,82 & aprox. zero & rejeitar $\mathrm{H} 0$ \\
\hline $\begin{array}{l}\text { Consumo de solução antisética } \\
\text { (litros) por } 1000 \text { doentes/dia }\end{array}$ & 23,55 & $23,34(11,13)$ & 48 & $0,1 \%$ & $-0,14$ & aprox. zero & não rejeitar H0 \\
\hline
\end{tabular}

(1): O valor de jan 2008; (2): Número de observações; (3): Nível de confiança. 
Incidência de MRSA por 1000 doentes/dia

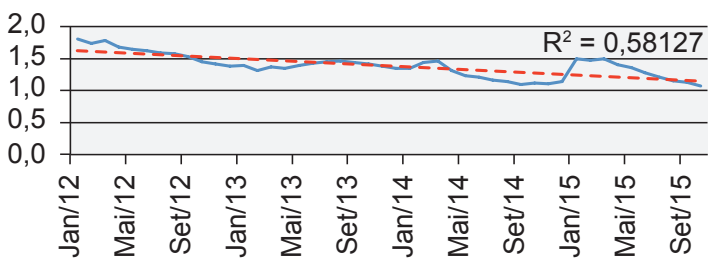

Consumo de carbapenemos em DDDs por 100 doentes/dia
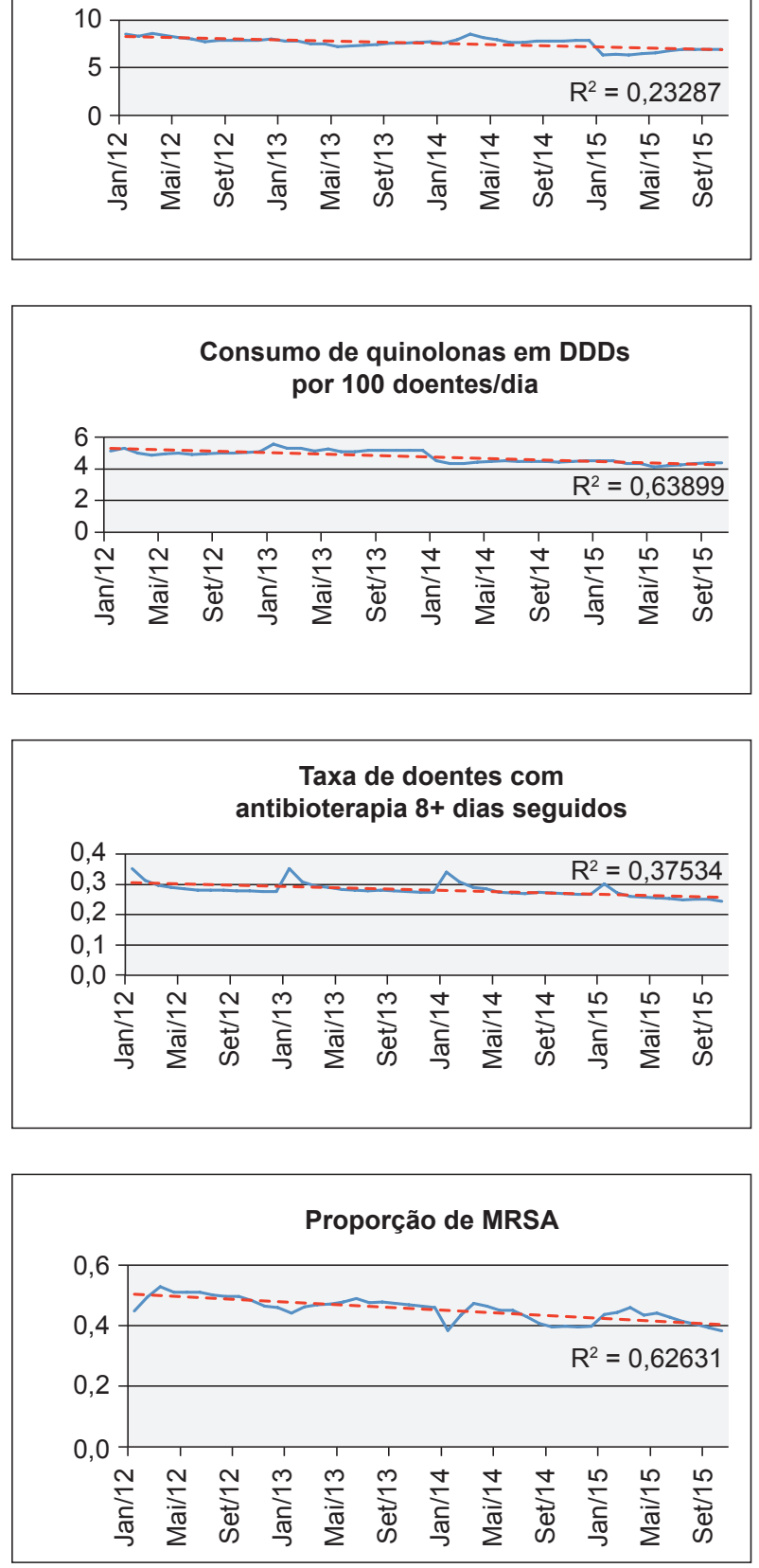

Figura 2 - Tendência dos parâmetros no período 2012 - 2015
Consumo de solução antiséptica (litros) por 1000 doentes/dia

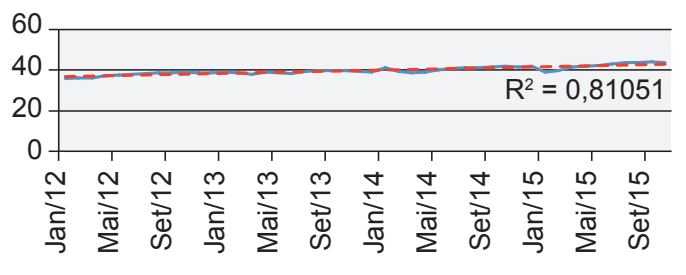

Incidência de microorganismos multi-resistentes por 1000 doentes/dia

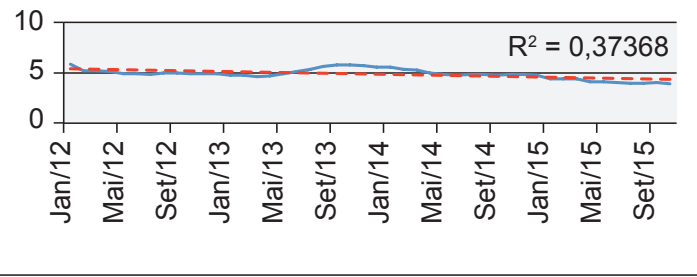

Consumo de antibióticos em DDDs por 100 doentes/dia
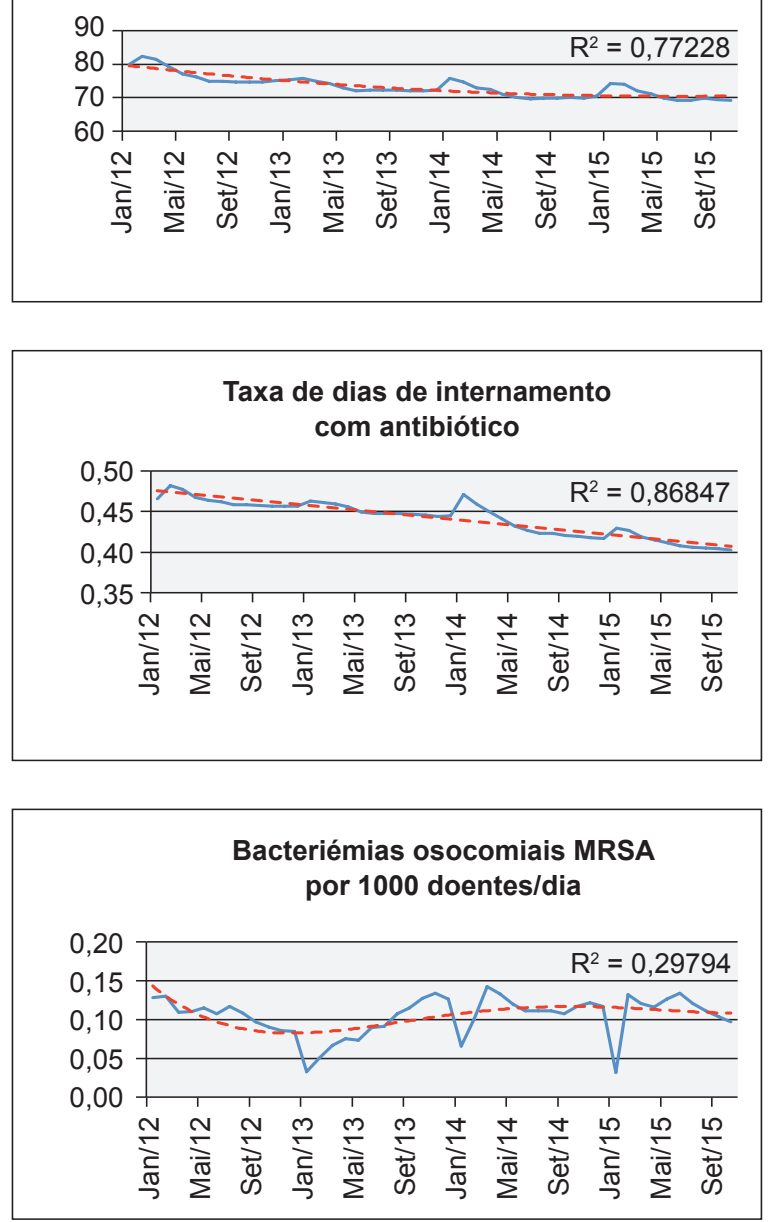
Tabela 2 - Teste de Hipóteses para a média (dados de 2012 a 2015)

\begin{tabular}{|c|c|c|c|c|c|c|c|}
\hline & $\mu_{0}{ }^{(1)}$ & Média e DP & $\mathrm{n}^{(2)}$ & $\alpha^{(3)}$ & Z observado & Valor $p$ & $\begin{array}{l}\text { Resultado do } \\
\text { teste }\end{array}$ \\
\hline $\begin{array}{l}\text { Consumo de antibióticos em } \\
\text { DDDs por } 100 \text { doentes/dia }\end{array}$ & 76,27 & $73,23(3,25)$ & 46 & $0,1 \%$ & $-6,35$ & aprox. zero & rejeitar HO \\
\hline $\begin{array}{l}\text { Consumo de carbapenemos em } \\
\text { DDDs por } 100 \text { doentes/dia }\end{array}$ & 9,11 & $7,60(0,58)$ & 46 & $0,1 \%$ & $-17,56$ & aprox. zero & rejeitar HO \\
\hline $\begin{array}{l}\text { Consumo de quinolonas em } \\
\text { DDDs por } 100 \text { doentes/dia }\end{array}$ & 5,17 & $4,77(0,39)$ & 46 & $0,1 \%$ & $-6,95$ & aprox. zero & rejeitar $\mathrm{H} 0$ \\
\hline $\begin{array}{l}\text { Taxa de dias de int com } \\
\text { antibiótico }\end{array}$ & 0,47 & $0,44(0,02)$ & 46 & $0,1 \%$ & $-7,63$ & aprox. zero & rejeitar $\mathrm{H} 0$ \\
\hline $\begin{array}{l}\text { Taxa de doentes com } \\
\text { antibioterapia } 8+\text { dias seguidos }\end{array}$ & 0,29 & $0,28(0,02)$ & 46 & $0,1 \%$ & $-3,19$ & aprox. zero & rejeitar $\mathrm{HO}$ \\
\hline $\begin{array}{l}\text { Consumo de solução antiséptica } \\
\text { (litros) por } 1000 \text { doentes/dia }\end{array}$ & 23,34 & $39,84(2,02)$ & 46 & $0,1 \%$ & 55,38 & aprox. zero & rejeitar HO \\
\hline $\begin{array}{l}\text { Incidência de microorganismos } \\
\text { multi-resistentes } 1000 \text { doentes/dia }\end{array}$ & 5,82 & $4,87(0,51)$ & 46 & $0,1 \%$ & $-12,58$ & aprox. zero & rejeitar HO \\
\hline $\begin{array}{l}\text { Incidência de MRSA por } 1000 \\
\text { doentes/dia }\end{array}$ & 1,80 & $1,38(0,19)$ & 46 & $0,1 \%$ & $-15,15$ & aprox. zero & rejeitar $\mathrm{H} 0$ \\
\hline Proporção de MRSA & 0,53 & $0,45(0,04)$ & 44 & $0,1 \%$ & $-13,22$ & aprox. zero & rejeitar HO \\
\hline $\begin{array}{l}\text { Bacteriémias nosocomiais MRSA } \\
\text { por } 1000 \text { doentes/dia }\end{array}$ & 0,13 & $0,10(0,03)$ & 46 & $0,1 \%$ & $-6,35$ & aprox. zero & rejeitar $\mathrm{H} 0$ \\
\hline
\end{tabular}

(1): Média 2008-2011; (2): Número de observações; (3): Nível de confiança.

Tabela 3 - Teste de Hipóteses para a média (dados de 2008 a 2011 vs 2012 a 2015)

\begin{tabular}{|c|c|c|c|c|c|c|c|c|}
\hline & \multicolumn{2}{|c|}{2008 a 2011} & \multicolumn{2}{|c|}{2012 a 2015} & \multirow[b]{2}{*}{$\mathbf{\alpha}^{(1)}$} & \multirow[b]{2}{*}{ Z observado } & \multirow[b]{2}{*}{ Valor $p$} & \multirow[b]{2}{*}{$\begin{array}{l}\text { Resultado do } \\
\text { teste }\end{array}$} \\
\hline & Média e DP & $n$ & Média e DP & $\mathrm{n}$ & & & & \\
\hline $\begin{array}{l}\text { Consumo de antibióticos em } \\
\text { DDDs por } 100 \text { doentes/dia }\end{array}$ & $76,27(2,59)$ & 48 & $73,23(3,25)$ & 46 & $0,1 \%$ & -5 & aprox. zero & rejeitar $\mathrm{HO}$ \\
\hline $\begin{array}{l}\text { Consumo de carbapenemos em } \\
\text { DDDs por } 100 \text { doentes/dia }\end{array}$ & $9,11(1,81)$ & 48 & $7,6(0,58)$ & 46 & $0,1 \%$ & $-5,5$ & aprox. zero & rejeitar $\mathrm{HO}$ \\
\hline $\begin{array}{l}\text { Consumo de quinolonas em } \\
\text { DDDs por } 100 \text { doentes/dia }\end{array}$ & $5,17(0,24)$ & 48 & $4,77(0,39)$ & 46 & $1,0 \%$ & $-5,97$ & aprox. zero & rejeitar $\mathrm{HO}$ \\
\hline $\begin{array}{l}\text { Taxa de dias de int com } \\
\text { antibiótico }\end{array}$ & $0,43(0,03)$ & 48 & $0,44(0,02)$ & 46 & $0,1 \%$ & 1,6 & aprox. zero & não rejeitar H0 \\
\hline $\begin{array}{l}\text { Taxa de doentes com } \\
\text { antibioterapia } 8+\text { dias seguidos }\end{array}$ & $0,29(0,02)$ & 48 & $0,28(0,02)$ & 46 & $0,1 \%$ & $-2,28$ & aprox. zero & não rejeitar H0 \\
\hline $\begin{array}{l}\text { Consumo de solução antiséptica } \\
\text { (litros) por } 1000 \text { doentes/dia }\end{array}$ & $23,34(11,13)$ & 48 & $39,84(2,02)$ & 46 & $0,1 \%$ & 10,1 & aprox. zero & não rejeitar H0 \\
\hline
\end{tabular}

(1): Nível de confiança

melhoria do consumo de SABA. No entanto, pela própria existência do sistema informático, os profissionais de saúde ficam mais sensibilizados e alertados para esta situação de risco, podendo, por este motivo, colaborar mais na prática das medidas básicas de controlo de infeção.

Em Portugal a qualidade da prestação de cuidados dos sistemas de saúde depara-se com novos desafios, não só pela necessidade de controlo dos custos no sector, mas também pelos diferentes problemas de saúde vivenciados pelos utentes. Assim, será fundamental a criação e implementação de políticas orientadas para novas estratégias de intervenção. Deve-se privilegiar uma abordagem integrada com o objetivo de melhorar progressivamente o nível de saúde das populações, a qualidade dos cuidados 

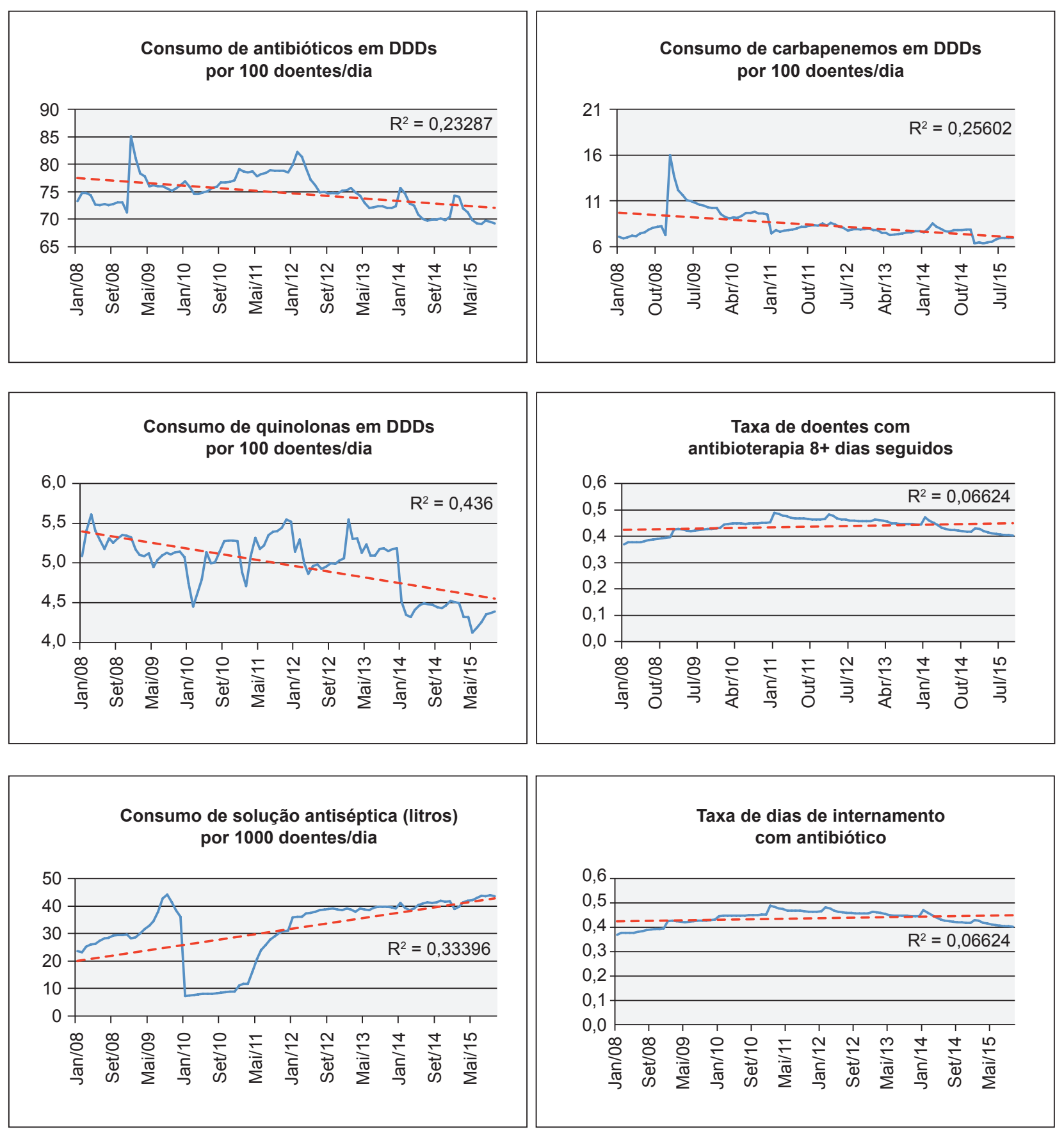

Figura 3 - Tendência dos parâmetros no período 2008 - 2015

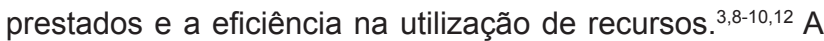
gestão clínica da doença centrada no doente desenvolve-se segundo as melhores práticas profissionais, visando, também, a uniformização. O HViTAL é um sistema de informação que permite monitorizar e avaliar constantemente esse processo.

\section{CONCLUSÃO}

Analisados os dados recolhidos, houve uma inequívoca melhoria em todos os domínios que caracterizam as IACS. É provável que o HViTAL facilite a vigilância das IACS, tenPROTECÇÃO DE PESSOAS E ANIMAIS

Os autores declaram que os procedimentos seguidos estavam de acordo com os regulamentos estabelecidos pelos responsáveis da Comissão de Investigação Clínica e Ética e de acordo com a Declaração de Helsínquia da Associação Médica Mundial.

\section{CONFIDENCIALIDADE DOS DADOS}

Os autores declaram ter seguido os protocolos do seu centro de trabalho acerca da publicação de dados. 


\section{CONFLITOS DE INTERESSE}

Os autores declaram não terem qualquer conflito de interesse relativamente ao presente artigo.

\section{REFERÊNCIAS}

1. Humphreys $\mathrm{H}$, Smyth ET. Prevalence surveys of healthcare - associated infections: what do they tell us, if anything? Clin Microbiol Infect. 2006;12:2-4

2. Pittet D, Allegranzi B, Storr J, Bagheri Nejad S, Dziekan G, Leotsakos A, et al. Infection control as a major World Health Organization priority for developing countries. J Hosp Infect. 2008;68:4:285-92.

3. Direção Geral da Saúde. Programa Nacional de Prevenção e Controlo da Infecção Associada aos Cuidados de Saúde, Vigilância Epidemiológica das Infecções Associadas aos Cuidados de Saúde, Critérios para Definição de Infecções nos Cuidados de Saúde Agudos. Lisboa: DGS; 2009.

4. Yu-Sheng L, Wen-Sen L, Guo-Bin C, Chien-Tsai L. Improving the work efficiency of healthcare-associated infection surveillance using electronic medical records. Comput Methods Programs Biomed. 2014;117:351-9.

5. Rochefort CM, Buckeridge DL, Forster AJ. Accuracy of using automated methods for detecting adverse events from electronic health record data: a research protocol. Implement Sci. 2015;10:5.

6. Hum RS, Cato K, Sheehan B, Patel S, Duchon J, DeLaMora P, et al. Developing clinical decision support within a commercial electronic health record system to improve antimicrobial prescribing in the neonatal ICU. Appl Clin Inform. 2014;4;5:368-87.

\section{FONTES DE FINANCIAMENTO}

Os autores declaram não ter recebido subsídios ou bolsas para a elaboração do artigo.

7. Pina E, Silva G, Ferreira E. Relatório do Inquérito de Prevalência de Infecção - Programa Nacional de Prevenção e Controlo da Infecção Associada aos Cuidados de Saúde. Lisboa: DGS; 2010.

8. Direcção Geral da Saúde. Prevalência de Infeção Adquirida no Hospital e do uso de Antimicrobianos nos Hospitais Portugueses. Inquérito 2012. Lisboa: DGS; 2012.

9. Direcção Geral da Saúde. Vigilância Epidemiológica das Resistências aos Antimicrobianos. Norma 004/2013. Lisboa: DGS; 2013.

10. Escoval A, Coelho A, Diniz JA, Rodrigues M, Moreira F, Espiga P. Gestão integrada da doença: uma abordagem experimental de gestão em saúde. Rev Port Saúde Pública. 2010;9:105-16.

11. Direcção Geral da Saúde. Terapêutica Antibiótica, Duração, Resistência a Antimicrobianos, Pressão antibiótica. Norma 006/2014 atualizada a 08/05/2015. Lisboa: DGS; 2015.

12. Pina E, Ferreira E, Marques A, Matos B. Infecções associadas aos cuidados de saúde e segurança do doente. Rev Port Saúde Pública. 2010;10:27-39.

13. Publichealthontario.ca [homepage na Internet]. Public Health Ontario: Antimicrobial Stewardship Programs. [consultado 2015 dez 12]. Disponivel em: http://www.publichealthontario.ca/en/eRepository/ ASP\%20Metric\%20examples.pdf. 